\title{
Avaliação da aptidão física de idosas em um Programa de Atividades Físicas
}

\author{
Evaluation of the physical fitness of elderly people in a Program of Physical Activities \\ Evaluación de la aptitud física de personas mayores en un Programa de Actividades Físicas
}

Recebido: 25/03/2021 | Revisado: 08/04/2021 | Aceito: 09/04/2021 | Publicado: 12/04/2021

\author{
Vitor Viana da Costa \\ ORCID: https://orcid.org/0000-0002-1639-1240 \\ Universidade Federal do Ceará, Brasil \\ E-mail: vitorg12ce@hotmail.com \\ André Accioly Nogueira Machado \\ ORCID: https://orcid.org/0000-0003-0838-2835 \\ Universidade Estadual do Ceará, Brasil \\ E-mail: andre.accioly@uece.br \\ Francisco Sérgio Lopes Vasconcelos-Filho \\ ORCID: https://orcid.org/0000-0003-3140-0085 \\ Universidade Federal do Cariri, Brasil \\ E-mail: sergio.vasconcelos@ufca.edu.br \\ Nielpson Dias Carvalho \\ ORCID: https://orcid.org/0000-0001-7095-5986 \\ Universidade Estadual do Ceará, Brasil \\ E-mail: nielpsoncarvalho@yahoo.com.br \\ Mabelle Maia Mota \\ ORCID: https://orcid.org/0000-0002-4265-7647 \\ Universidade Estadual do Ceará, Brasil \\ E-mail: mabellemota@gmail.com \\ Welton Daniel Nogueira Godinho \\ ORCID: https://orcid.org/0000-0001-5047-9273 \\ Universidade Estadual do Ceará, Brasil \\ E-mail: welton.daniel@uece.br \\ Paula Matias Soares \\ ORCID: https://orcid.org/0000-0002-3592-4444 \\ Universidade Estadual do Ceará, Brasil \\ E-mail: paula.soares@uece.br \\ Alex Soares Marreiros Ferraz \\ ORCID: https://orcid.org/0000-0002-2331-7698 \\ Universidade Federal do Ceará, Brasil \\ E-mail: ferrazalex@hotmail.com
}

\section{Resumo}

Atrelado a um bom programa de atividades físicas, geralmente, têm-se métodos para avaliar as capacidades funcionais de indivíduos que dele participam. As informações geradas por esta avaliação possibilitam conhecer as características dos idosos a partir de ferramentas simples e úteis, auxiliando no diagnóstico das limitações específicas de cada indivíduo e na definição de estratégias para promoção de saúde, visando retardar ou prevenir as incapacidades resultantes do envelhecimento. Assim, o objetivo desta pesquisa foi analisar, em diferentes grupos etários, os níveis de aptidão física funcional de idosas participantes de um programa de atividades físicas. O estudo realizado foi de natureza quantitativa e característica descritiva. A pesquisa foi realizada no município do Crato-Ceará, na qual participaram 47 idosas integrantes do programa Saúde, Bombeiros e Sociedade. Para a coleta dos dados, foi aplicado um questionário e realizada uma avaliação antropométrica e uma avaliação da aptidão física, esta mensurada através da bateria de testes de a Rikli e Jones. Percebeu-se que todas apresentaram resultados abaixo da média para o teste "Caminhada de seis minutos"; a faixa etária com o maior número de testes abaixo da média foi a entre 60 e 64 anos, seguida pela faixa etária de 74 a 79 anos, com dois testes. Na análise geral dos resultados, percebe-se que, quanto à resistência aeróbica, agilidade e equilíbrio dinâmico, as participantes demonstraram resultados insatisfatórios ou abaixo da média, indicando um déficit nessas valências.

Palavras-chave: Aptidão física; Exercício Físico; Qualidade de vida; Idoso.

\footnotetext{
Abstract

Linked to a good physical activity program, there are usually methods to assess the functional capacities of individuals who participate in it. The information generated by this evaluation makes it possible to know the characteristics of the elderly from simple and useful tools, assisting in the diagnosis of the specific limitations of each individual and in the definition of strategies for health promotion, aiming to delay or prevent the disabilities resulting
} 
from aging. Thus, the objective of this research was to analyze, in different age groups, the levels of functional physical fitness of elderly women participating in a physical activity program. The study was quantitative and descriptive. The research was carried out in the municipality of Crato - Ceará, in which 47 elderly members of the Health, Fire and Society program participated. For data collection, a questionnaire was applied and an anthropometric assessment and an assessment of physical fitness was carried out, this measured through the battery of tests by Rikli and Jones. It was noticed that all of them presented below average results for the "Six-minute walk" test; the age group with the highest number of tests below the average was between 60 and 64 years, followed by the age group of 74 to 79 years, with two tests. In the general analysis of the results, it can be seen that, regarding aerobic endurance, agility and dynamic balance, the participants showed unsatisfactory results or below average, indicating a deficit in these valences.

Keywords: Physical Aptitude; Physical Exercise; Quality of Life; Elderly.

\section{Resumen}

Vinculado a un buen programa de actividad física, suelen existir métodos para evaluar las capacidades funcionales de las personas que participan en él. La información generada por esta evaluación permite conocer las características de las personas mayores a partir de herramientas sencillas y útiles, ayudando en el diagnóstico de las limitaciones específicas de cada individuo y en la definición de estrategias de promoción de la salud, con el objetivo de retrasar o prevenir las discapacidades. resultante del envejecimiento. Así, el objetivo de esta investigación fue analizar, en diferentes grupos de edad, los niveles de aptitud física funcional de mujeres mayores que participan en un programa de actividad física. El estudio fue cuantitativo y descriptivo. La investigación se llevó a cabo en el municipio de Crato - Ceará, en la que participaron 47 ancianos miembros del programa Salud, Bomberos y Sociedad. Para la recogida de datos se aplicó un cuestionario y se realizó una valoración antropométrica y una valoración de la aptitud física, que se midió mediante la batería de pruebas de Rikli y Jones. Se notó que todos presentaron resultados por debajo del promedio para la prueba de "Caminata de seis minutos"; el grupo de edad con mayor número de pruebas por debajo de la media fue entre 60 y 64 años, seguido del grupo de edad de 74 a 79 años, con dos pruebas. En el análisis general de los resultados, se puede observar que, en cuanto a resistencia aeróbica, agilidad y equilibrio dinámico, los participantes mostraron resultados insatisfactorios o por debajo de la media, lo que indica un déficit en estas valencias. Palabras clave: Aptitud Física; Ejercicio Físico; Calidad de Vida; Anciano.

\section{Introdução}

O envelhecimento pode ser considerado um fenômeno natural, capaz de provocar transformações fisiológicas, psicológicas e sociais em seres vivos. Este fenômeno, quanto à população, vem sendo considerado um desafio para a saúde pública, devido à velocidade com que progride (Fechine \& Trompieri, 2012; Pedreira et al., 2016).

Com o envelhecimento, comumente, surgem as perdas funcionais, devido aos efeitos inerentes a este processo sobre o organismo. Entretanto, seus efeitos variam de um indivíduo para o outro, apresentando-se, para alguns, como um processo lento e, para outros, mais veloz (Pilger et al, 2013; Caetano, 2006).

Assim sendo, passa-se a adotar, como principais indicadores de saúde na população idosa, não mais a presença ou ausência de doenças e, sim, os graus de capacidades físicas, evidenciados, por exemplo, a partir da diminuição da força física e da falta de equilíbrio (Bezerra, Almeida \& Nobrega-Therrien, 2012; Ramos, 2009)

Contrariamente a isto, manter bons níveis na aptidão física na melhor idade, pode representar melhorias tanto nos aspectos físicos como funcionais, demonstrando avanços em relação a composição corporal, controle de peso, capacidades funcionais e condicionamento físico, estando estes diretamente ligados à saúde desta população (Costa, Soares, Gomes \& Machado, 2018).

Algumas ferramentas tornam-se imprescindíveis para ganhos ou manutenção da aptidão física, e, neste rol de possibilidades, tem-se a prática de atividades físicas, como uma forma de promover a saúde, protegendo as capacidades funcionais, independentemente da idade de quem as pratica. Porém, ao idoso, estas práticas tornam-se ainda mais importantes, pela contribuição para um envelhecimento com maior qualidade (Fraccari, Piccoli \& Quevedo, 2012; Vila et al., 2013).

Como uma estratégia eficaz, a literatura evidencia a participação em programas de atividades físicas, a qual pode ser fundamental para a saúde de idosos. Esta participação, é responsável por melhorias nos aspectos físicos e ir além, favorecendo um acompanhamento mais estreito entre o profissional e o praticante, além da interação com outros participantes, promovendo 
evoluções quanto à interação social, autoestima, e sensação de autocontrole (Morgado, 2013; Mazo, Lopes \& Beneditti, 2009; Ribeiro et al., 2009).

Atrelado a um bom programa de atividades físicas, geralmente, têm-se métodos para avaliar as capacidades funcionais de indivíduos que dele participam. As informações geradas por esta avaliação possibilitam conhecer as características dos idosos a partir de ferramentas simples e úteis, auxiliando no diagnóstico das limitações específicas de cada indivíduo e na definição de estratégias para promoção de saúde, visando retardar ou prevenir as incapacidades resultantes do envelhecimento (Barbosa et al., 2014; Xavier et al., 2000).

$\mathrm{Na}$ literatura brasileira, há um déficit no que tange pesquisas acerca de programas de que promovam atividades e exercícios físicos para idosos (Squarcini et al., 2015), Contanto, esta pesquisa se baseia e se justifica pela necessidade do desenvolvimento de estudos que envolvem qualidade de vida e aptidão física funcional em idosos participantes destes programas, enfatizando a importância de um devido acompanhamento durante a prática de atividades físicas desta população, Assim, o objetivo desta pesquisa foi analisar, em diferentes grupos etários, os níveis de aptidão física funcional de idosas participantes de um programa de atividades físicas.

\section{Metodologia}

$\mathrm{O}$ estudo realizado foi de natureza quantitativa e característica descritiva. Esta pesquisa, de campo, foi realizada em praças, parques e espaços públicos do município do Crato, no estado do Ceará, onde era confirmada a presença do programa Saúde, Bombeiros e Sociedade.

Participaram do estudo 47 idosas que frequentavam regularmente do programa Saúde, Bombeiros e Sociedade da cidade do Crato e seguiram os seguintes critérios de inclusão: a) participar das aulas do programa Saúde, Bombeiros e Sociedade, por, pelo menos, duas vezes durante a semana; b) estar há mais de três meses inclusas no programa; c) não apresentar restrições à prática de atividade física. Foram excluídas da pesquisa as idosas que: a) não compareceram a todos os dias de testes de aptidão física; b) tinham problemas osteoarticulares ou outro que comprometesse a execução dos testes.

A pesquisa foi realizada em conformidade com a resolução nº466/12 do Conselho Nacional de Saúde (Brasil, 2012) que estabelece a eticidade da pesquisa em: autonomia, beneficência, não maleficência, justiça e equidade. Todavia, as participantes receberam e assinaram o termo de consentimento livre e esclarecido. Vale ressaltar que o processo de coleta dos dados destas participantes é parte integrada ao programa aqui estudado, sendo uma das ações desenvolvida por este.

Para a coleta dos dados, foi utilizado um questionário composto por perguntas acerca de dados sociodemográficos, assim como a frequência com que as idosas vão à praça para realizar as atividades do programa, dentre outras. Refere-se também ao programa de Saúde, Bombeiro e Sociedade, onde foram obtidas informações referentes ao programa.

Em seguida, foram realizadas uma avaliação antropométrica e uma avaliação da aptidão física. Nesta avaliação, foram realizadas as medidas de massa corporal e estatura, para cálculo do índice de massa corporal (IMC) e Circunferência da Cintura e do Quadril.

Para aferir a massa corporal, foi utilizada uma balança digital com precisão de 100 gramas. Para a medida da estatura foi utilizado estadiômetro portátil da marca Sanny. O IMC foi calculado a partir da divisão da massa corporal (quilogramas) pelo quadrado da estatura (metros). Na seguinte equação: $\mathrm{IMC}=\mathrm{kg} / \mathrm{m}^{2}$. Os valores considerados como ponto de corte são os referenciados pela Organização Mundial de Saúde (OMS) (Who, 2000).

Em seguida, foram realizadas as medidas de circunferência da cintura e quadril, com o auxílio de uma fita métrica, não elástica, de marca Sanny, com precisão de $1 \mathrm{~mm}$. Os pontos de corte para o risco cardiovascular relacionado à Circunferência da Cintura isolada foram os seguintes: para as mulheres, baixo risco $<80 \mathrm{~cm}$, risco elevado $\geq 80 \mathrm{~cm}$ e risco muito 
elevado $\geq 88 \mathrm{~cm}$ (Who, 2000). Após a medida da circunferência do quadril foi calculada, junto à circunferência da cintura, a Relação Cintura-Quadril (RCQ) (os valores considerados como ponto de corte para o risco cardiovascular relacionado à RCQ estão de acordo com a classificação sugerida por Heyward e Stolarczik (2000).

A aptidão física foi mensurada através da bateria de testes de aptidão física funcional desenvolvida por Rikli e Jones (1999). Os testes realizados e seus respectivos objetivos foram os seguintes: a) Sentar e Levantar em 30 segundos (avaliar força e resistência de membros inferiores); b) Flexão de cotovelo com halteres em 30 segundos (avaliar força e resistência dos membros superiores); c) Caminhada de seis minutos (avaliar a capacidade aeróbica); d) Sentar e Alcançar na Cadeira (avaliar flexibilidade de tronco e dos membros inferiores); e) Alcançar mãos às Costas (avaliar a flexibilidade dos membros superiores) e; f) Levantar e Caminhar (avaliar a agilidade e equilíbrio dinâmico).

Os resultados dos testes aplicados foram comparados aos valores de referência apresentados por Rikli e Jones (2001), classificando cada um destes "Abaixo da média"; "Na Média" ou "Acima da Média". A análise dos dados foi feita através de um estudo estatístico descritivo, descrevendo os dados de três maneiras: por meio de tabelas, de gráficos e de medidas descritivas expressas com média, desvio padrão, valor mínimo e valor máximo (Guedes, Martins, Acorsi e Janeiro). Os dados foram organizados e analisados no Excel® 2010 (Microsoft).

\section{Resultados e Discussão}

Neste tópico, foram apresentados os resultados referentes ao perfil sociodemográfico, dados referentes à participação no programa, resultados da avaliação antropométrica e dos resultados da aplicação dos testes indicados para a pesquisa.

Na Tabela 1, foram descritos os dados quanto a idade, estado civil, escolaridade e ocupação, traçando, assim, o perfil sociodemográfico das participantes desta pesquisa.

Tabela 1 - Descrição dos dados sociodemográficos das idosas participantes do projeto Bombeiros, saúde e Sociedade do município do Crato $-\mathrm{CE}(\mathrm{n}=47)$.

\begin{tabular}{cccc}
\hline \multicolumn{4}{c}{ Dados sociodemográficos } \\
\hline Variáveis & & \% & n \\
\hline \multirow{3}{*}{ Faixa etária } & $60-69$ & 59,6 & 28 \\
& $70-79$ & 27,7 & 13 \\
& 80 ou mais & 12,8 & 6 \\
\hline \multirow{3}{*}{ Estado civil } & Solteira & 19,1 & 9 \\
& Casada & 40,4 & 19 \\
& Viúva & 40,4 & 19 \\
\hline \multirow{3}{*}{ Escolaridade } & Analfabeta & 8,5 & 4 \\
& Fundamental & 61,7 & 29 \\
& Médio & 21,3 & 10 \\
& Superior & 8,5 & 4 \\
\hline \multirow{2}{*}{ Ocupação } & Dona de casa & 70,5 & 31 \\
& Aposentada & 15,9 & 7 \\
& Outras ocupações & 13,6 & 6 \\
\hline
\end{tabular}

Fonte: Autores.

Sobre os achados acerca da faixa etária, Cipriani, Meurer, Benedetti e Lopes (2010) e Ribeiro et al (2015) descrevem resultados semelhantes, onde a maior parte participante de programas de atividades físicas é formada por indivíduos com idade entre 60 e 69 anos. De acordo com Brasil (2013), no Brasil, a população tende a diminuir a frequência com que prática de atividades físicas, no tempo livre, com o aumento da idade, o que pode explicar os resultados aqui vistos. 
Quanto ao estado civil, houve igualdade no número de participantes casadas e viúvas. Entretanto, nas pesquisas consultadas, houve predominância para aquelas que vivem sua viuvez (Gomes \& Zazá, 2009; Sousa \& Silver, 2008) e tal acontecimento confirma o que foi dito por Paschoal (2004), quando afirma que mulheres idosas têm maior longevidade.

Quando observados os valores sobre escolaridade, nota-se que aquelas que cursaram somente o fundamental é predominante, resultado bastante aproximado ao encontrado por Cipriani et al (2010) e Binotto, Borgatto e Farias (2010). Assim como os resultados relacionados à ocupação das idosas entrevistadas, que mostram que a grande maioria se autointitula como donas de casas, estes resultados podem ter como principal causa, de acordo com Inouye e Pedrazzani (2007), o fato de que, décadas atrás, a sociedade não priorizava a educação escolar de mulheres e que, por vezes, estas eram criadas apenas para serem boas esposas e donas de casa.

$\mathrm{Na}$ Tabela 2, estão descritas as informações referentes ao programa de exercício como, tempo e motivação, e, informação sobre ter alcançado ou não seus objetivos no programa.

Tabela 2 - Descrição dos dados relacionados ao programa das idosas participantes do projeto Bombeiros, saúde e Sociedade do município do Crato $-\mathrm{CE}(\mathrm{n}=47)$.

\begin{tabular}{|c|c|c|c|}
\hline \multicolumn{4}{|c|}{ Dados relacionados ao programa } \\
\hline Variáveis & & $\%$ & $\mathbf{N}$ \\
\hline \multirow{3}{*}{$\begin{array}{l}\text { Motivos para } \\
\text { participar }\end{array}$} & Saúde & 87,2 & 41 \\
\hline & Socialização & 28,8 & 30 \\
\hline & Qualidade de vida & 21,2 & 22 \\
\hline \multirow{3}{*}{$\begin{array}{l}\text { Tempo que participa } \\
\text { do programa (meses) }\end{array}$} & Até 12 & 14,8 & 7 \\
\hline & $12-24$ & 34 & 16 \\
\hline & Acima de 24 & 51 & 24 \\
\hline \multirow{2}{*}{ Frequência semanal } & 2 dias/semana & 93,6 & 44 \\
\hline & 3 dias /semana & 6,4 & 3 \\
\hline \multirow{3}{*}{$\begin{array}{c}\text { Objetivos pessoais } \\
\text { atingidos }\end{array}$} & Sim & 93,6 & 44 \\
\hline & Não & 2,1 & 1 \\
\hline & Parcialmente & 4,3 & 2 \\
\hline
\end{tabular}

Fonte: Autores.

Os achados de Batista e Almeida (2020) corroboram para os dados relatados acima, afirmando que a saúde é o fator principal para a procura pelos programas de atividades físicas e que, em maioria, as idosas participam deste cerca de duas vezes por semana. Meurer, Benedetti e Mazo (2012), justificam que, as questões voltadas à saúde são as principais razões, tanto para entrar como para permanecer em um programa de atividades físicas para idosos, o que também é de explicação cabível para os resultados referentes ao tempo de permanência no programa e para o alcance dos objetivos (melhorar a saúde).

Na Tabela 3, estão descritas as médias e o desvio padrão (DP) das variáveis analisadas nesta pesquisa.

Tabela 3 - Descrição das médias referentes à avaliação antropométrica das idosas participantes do projeto Bombeiros, saúde e Sociedade do município do Crato - CE (n=47).

\begin{tabular}{cccccccc}
\hline & $\begin{array}{c}\text { Idade } \\
\text { (anos) }\end{array}$ & $\begin{array}{c}\text { Massa } \\
\text { corporal } \\
(\mathbf{K g})\end{array}$ & $\begin{array}{c}\text { Altura } \\
(\text { metros })\end{array}$ & $\begin{array}{c}\text { IMC } \\
(\mathbf{K g} / \mathbf{m} 2)\end{array}$ & $\mathbf{C C}(\mathbf{c m})$ & $\mathbf{C Q}(\mathbf{c m})$ & RCQ \\
\hline Média & 69,2 & 62 & 1,5 & 27,2 & 95,8 & 100,0 & 1,0 \\
DP & 7,3 & 8,7 & 0,1 & 3,9 & 10,0 & 10,0 & 0,1 \\
\hline
\end{tabular}

Fonte: Autores. 
Alguns estudos na literatura corroboram com os resultados descritos na tabela 03, apresentando, quando não iguais, bastante aproximados, como, por exemplo, Leite-Cavalcanti et al (2009), Miranda e Rabelo (2006) e Cabrera, Wajngarten, Gebara e Diament (2005) que encontram resultados semelhantes quanto as médias de idade, altura, CC, e RCQ das idosas estudas.

Considerando as indicações para Who (2000), percebe-se que a média do IMC representa que as pesquisadas estão acima do peso ideal, tendo, assim, perfil indicativo para sobrepeso (56\%), enquanto apenas $28,3 \%$ encontram-se com peso ideal para suas condições. Estes resultados foram semelhantes àqueles encontrados por Cunha et al (2010).

Entretanto, para Cyarto, Brown, Marshall e Trost (2008), os resultados podem ser explicados pelo fato de que as mulheres idosas tendem a ter uma diminuição na estatura e aumento de peso, incidindo, diretamente, sobre o IMC destas. Outro fator agravante é que idosas tendem a acumular mais gordura visceral e apresentar uma redistribuição da gordura dos membros para o abdômen, podendo resultar em sobrepeso ou obesidade (Who, 1995). Vale ressaltar a importância da manutenção do peso ideal, pois torna-se, inclusive, fundamental na prevenção de doenças crônicas.

Para Loureiro et al. (2020), os indicadores antropométricos aqui utilizados são de grande relevância para a identificação de fatores de risco para doenças cardiovasculares, alegando a importância de utilizá-los em estudos, no caso, com idosos. Além disso, enaltecem-nos por serem métodos simples, de baixo custo e não invasivos, "contribuindo para a identificação precoce dos fatores de risco, possibilitando ações e estratégias de prevenção e controle das doenças cardiovasculares" (Loureiro et al., 2020, p. 12).

$\mathrm{Na}$ Tabela 4, estão representados os resultados de cada teste com os dados referentes às médias, desvio padrão e as classificações, divididos por idade. 
Tabela 4 - Descrição dos resultados das aplicações dos testes de Rikli e Jones (1999), divididos por faixa etária, das participantes do projeto Bombeiros, saúde e Sociedade do município do Crato - CE (n=47).

\begin{tabular}{|c|c|c|c|c|c|c|}
\hline & $\begin{array}{l}\text { Caminhada } \\
\text { de seis } \\
\text { minutos } \\
\text { (metros) }\end{array}$ & $\begin{array}{c}\text { Sentar e } \\
\text { levantar } \\
\text { em 30" } \\
\text { (rep) }\end{array}$ & $\begin{array}{l}\text { Flexão de } \\
\text { cotovelo } \\
\text { em 30" } \\
\text { (rep) }\end{array}$ & $\begin{array}{l}\text { Sentar e } \\
\text { alcançar } \\
\text { na } \\
\text { cadeira } \\
(\mathrm{cm})\end{array}$ & $\begin{array}{c}\text { Alcançar } \\
\text { mãos às } \\
\text { costas } \\
(\text { cm) }\end{array}$ & $\begin{array}{c}\text { Levantar } \\
\text { e } \\
\text { Caminhar } \\
(\text { seg })\end{array}$ \\
\hline \multicolumn{7}{|l|}{$\begin{array}{l}60-64 \\
(n=18)\end{array}$} \\
\hline Média & 371,7 & 14,3 & 15,4 & $-0,6$ & $-8,1$ & 6,8 \\
\hline DP & 105,7 & 4,6 & 3,7 & 10,8 & 12,7 & 1,3 \\
\hline Classificação & Abaixo & Média & Média & Média & Abaixo & Abaixo \\
\hline \multicolumn{7}{|l|}{$\begin{array}{l}65-69 \\
(n=10)\end{array}$} \\
\hline Média & 368,2 & 14,9 & 15,2 & 2,4 & $-7,3$ & 6,4 \\
\hline DP & 159,8 & 3,4 & 1,1 & 3,7 & 10,9 & 1,0 \\
\hline Classificação & Abaixo & Média & Média & Média & Média & Média \\
\hline \multicolumn{7}{|l|}{$\begin{array}{l}70-74 \\
(n=08)\end{array}$} \\
\hline Média & 426,9 & 13,5 & 13,3 & 1,4 & $-6,3$ & 6,9 \\
\hline DP & 67,3 & 2,7 & 2,7 & 3,5 & 7,9 & 1,2 \\
\hline Classificação & Abaixo & Média & Média & Média & Média & Média \\
\hline \multicolumn{7}{|l|}{$\begin{array}{l}75-79 \\
(n=05)\end{array}$} \\
\hline Média & 370,8 & 13,6 & 15,2 & 3,4 & $-11,2$ & 8,0 \\
\hline $\mathrm{DP}$ & 63,4 & 4,9 & 3,6 & 4,3 & 7,5 & 2,2 \\
\hline Classificação & Abaixo & Média & Média & Média & Média & Abaixo \\
\hline \multicolumn{7}{|l|}{$\begin{array}{l}80-84 \\
(n=06)\end{array}$} \\
\hline Média & 335,83 & 12,80 & 10,17 & 4,17 & $-12,00$ & 7,75 \\
\hline $\mathrm{DP}$ & 140,28 & 3,90 & 5,23 & 2,32 & 5,69 & 0,79 \\
\hline Classificação & Abaixo & Média & Média & Média & Média & Média \\
\hline \multicolumn{7}{|l|}{ Total $(n=47)$} \\
\hline Média & 375,7 & 14,0 & 14,3 & 1,4 & $-8,4$ & 7,0 \\
\hline $\mathrm{DP}$ & 113,9 & 3,9 & 3,7 & 7,3 & 10,2 & 1,4 \\
\hline Classificação & Abaixo & Média & Média & Média & Média & Abaixo \\
\hline
\end{tabular}

Fonte: Autores.

Após análise, percebeu-se que todas as idosas participantes apresentaram resultados abaixo dos esperados para o teste "Caminhada de seis minutos". A faixa etária com o maior número de testes abaixo da média, totalizando três, foi a entre 60 e 64 anos, seguida pela faixa etária de 74 a 79 anos, com dois testes. Entretanto, na literatura, encontram-se resultados dentro da média para este mesmo teste (Furtado, Pereira, Moreira \& Dantas, 2008; Nunes \& Santos, 2009).

O “caminhada de seis minutos" é um teste bastante simples e de baixo ou nenhum custo, capaz de possibilitar análises cardiorrespiratórias e metabólicas essenciais, facilitando tais diagnósticos. Quando um idoso apresenta resultados ruins, no caso, para o teste "caminhada de seis minutos", é preocupante, já que este, como o nome remete, é realizado a partir de uma atividade inerente ao ser humano, exceto aqueles afetados por quadros específicos (Morales-Blanhir et al., 2011; Li et al., 2005; Ats, 2002).

Assim, considerando o contexto desta pesquisa, pode-se considerar relevante incluir a caminhada aos programas de atividades físicas, sabendo que esta é capaz de promover benefícios importantes para a saúde, diminuindo riscos de morte, 
principalmente, por doenças crônico-degenerativas (Bicalho, Géa-Horta, Moreira, Gazzinelli \& Velasquez-Melendez, 2018; Who, 2011).

Um segundo teste que apresentou, recorrentemente, resultados abaixo dos esperados, foi o teste de Levantar e Caminhar, o qual identifica dificuldades relacionadas à agilidade e ao equilíbrio dinâmico. Na pesquisa de Martins (2016), neste teste, as idosas apresentaram uma média de 6,9 segundos, aproximado, porém, ainda abaixo do aqui mostrado. Apesar do equilíbrio e da agilidade diminuir com o envelhecimento, a prática regular de atividades físicas é capaz de reduzir sua perda, baseando-se em comparações entre pessoas idosas que as praticam e que não as praticam. A autora ainda afirma que "fica claro que a participação em programas de atividade física é uma forma eficaz de reduzir e prevenir diversos declínios funcionais comuns ao avançar da idade" (Silva, 2017, p. 85).

Para Miyasike-da-Silva, Villar, Zago, Polastri \& Gobbi (2002), a agilidade é de grande valia nas atividades da vida diária (AVDs), devido às necessidades de deslocar-se e desviar-se de pessoas e objetos durante seus trajetos, contribuindo, também, para a manutenção da qualidade de vida. Hauser et al (2015), ressaltam, também, a necessidade de se trabalhar exercícios que provoquem o desenvolvimento do equilíbrio dinâmico, por esta, assim como a agilidade, "ser uma ferramenta essencial para a melhora da qualidade de vida e a percepção da população idosa em relação à sua autonomia e independência em tarefas do cotidiano" (Hauser et al., 2015, p. 275).

\section{Considerações Finais}

A partir dos resultados, percebeu-se que as participantes do Programa Saúde, Bombeiros e Sociedade da cidade do Crato-CE apresentaram um perfil antropométrico alterado, resultando em um maior percentual para o perfil de "Sobrepeso", ou seja, acima do peso ideal. Além disso, estas afirmam que seus objetivos, ao participar do programa de atividades físicas, eram voltados para a busca pela recuperação ou manutenção da saúde;

As idosas participantes com idade entre 60 e 64 anos foram as que mais deixaram a desejar em seus resultados, com metade dos testes abaixo da média (nos testes Caminhada de seis minutos", "Alcançar Mãos às Costas" e "Levantar e Caminhar"), demonstrando baixa capacidade aeróbica, dificuldades com a flexibilidade dos membros superiores e com agilidade e equilíbrio dinâmico.

Já as participantes com idades entre 75 e 79 anos, a maior parte dos resultados alcançaram a média (cerca de 65\%), demonstrando bons resultados referentes à força e resistência dos membros superiores e inferiores, e flexibilidade dos membros superiores, inferiores e de tronco. Entretanto, os resultados que avaliaram a capacidade aeróbica e a agilidade e equilíbrio dinâmico (teste de Caminhada de minutos e Levantar e Caminhar) apresentaram resultados abaixo da média.

As participantes com idades entre 65 e 74 anos e 80 a 84 anos apresentaram os melhores resultados para a Aptidão Física, ficando com 83,25\% dos resultados acima da média, ficando abaixo da média apenas no teste de Caminhada de seis minutos. Na análise geral dos resultados, percebe-se que, quanto à resistência aeróbica, todas as participantes demonstraram resultados insatisfatórios ou abaixo da média, indicando um déficit nesta valência.

\section{Referências}

Ats. (2002). Committee on Proficiency Standards for Clinical Pulmonary Function Laboratories. ATS statement: guidelines for the six-minute walk test. Am $J$ Respir Crit Care Med, 166(1), 111-7. https://doi.org/10.1164/ajrccm.166.1.at1102.

Batista, R. A. \& Almeida, E. A. (2020). Motivos para a adesão e permanência do idoso nas atividades de hidroginástica. Trabalho de Conclusão de Curso (Graduação em Educação Física) - Centro Universitário São Lucas - UniSL, 2020.

Barbosa, B. R., Almeida, J. M., Barbosa, M. R. \& Barbosa, L. A. R. R. (2014). Avaliação da capacidade funcional dos idosos e fatores associados à incapacidade. Ciência \& Saúde Coletiva, 19(8), 3317-3325. https://doi.org/10.1590/1413-81232014198.06322013. 
Bezerra, F. C., Almeida, M. I. \& Nóbrega-Therrien, S. M. (2012). Estudos sobre envelhecimento no Brasil: revisão bibliográfica. Revista Brasileira de Geriatria e Gerontologia, 15(1),155-167. https://doi.org/10.1590/S1809-98232012000100017.

Bicalho, P. G., Géa-Horta, T., Moreira, A.D., Gazzinelli, A. \& Velasquez-Melendez, G. (2018). Associação entre fatores sociodemográficos e relacionados à saúde com a prática de caminhada em área rural. Ciênc. saúde colet, 23(4), 1323-1332. https://doi.org/10.1590/1413-81232018234.18242016.

Binotto, M. A., Borgatto, A. F. \& Farias, S. F. (2010). Nível de atividade física: questionário internacional de atividades físicas e tempo de prática em mulheres idosas. Rev. Bras. Geriatr. Gerontol, 13(3), 425-434. https://doi.org/10.1590/S1809-98232010000300009.

Brasil. (2012). Conselho Nacional de Saúde. Resolução nº 466, de 12 de dezembro de 2012. Brasília.

Brasil. (2013). Vigitel Brasil 2009: vigilância de fatores de risco e proteção para doenças crônicas por inquérito telefônico. Ministério da Saúde, Secretaria de Vigilância em Saúde, Secretaria de Gestão Estratégica e Participativa, Brasília, DF, Brasil.

Cabrera, M. A. S., Wajngarten, M., Gebara, O. C. E., \& Diament, J. (2005). Relação do índice de massa corporal, da relação cintura-quadril e da circunferência abdominal com a mortalidade em mulheres idosas: seguimento de 5 anos. Cad Saúde Pública, 21(3), 767-775. https://doi.org/10.1590/S0102311 X2005000300010.

Caetano, L. M. (2006). O Idoso e a Atividade Física. Horizonte: Revista de Educação Física e desporto, 11(124), 20-28.

Cipriani, N. C. S., Meurer, S. T., Benedetti, T. R. B. \& Lopes, M. A. (2010) Aptidão funcional de idosas praticantes de atividades físicas. Rev Bras Cineantropom Desempenho Hum, 12(2),106-111. https://doi.org/10.5007/1980-0037.2010v12n2p106.

Costa, V.V., Soares, L. R., Gomes, A. N. \& Machado, A. A. N. (2018). Avaliação da Aptidão Física Funcional em Idosos e Implicações Para a Prescrição de Exercícios. Atuação Profissional em Educação e Saúde: Conceitos e Procedimentos. Curitiba: CRV, 2018. Coedição: Fortaleza, CE. Editora da Universidade Estadual do Ceará.

Cunha, H. A. V., Alves Júnior, A. J. T., Scabello, H. P. L., Figueiredo, R. R., Oliveira, I. L. G. \& Costa, J. B. (2010). Associação da obesidade à presença de comorbidades decorrentes em trabalhadores do Hospital e Maternidade Celso Pierro. Rev. Ciênc. Méd., 19(1-6), 23-31.

Cyarto, E. V. Brown, W. J., Marshall, A. L. \& Trost, S. G. (2008). Comparison of the effects of a homebased and group-based resistance training program on functional ability in older adults. American journal of health promotion, 23(1),13-17. https://doi.org/10.4278/ajhp.07030120.

Furtado, H. L., Pereira, F. D., Moreira, M. H. R. \& Dantas, E. H. M. (2008). Perfil da Resistência Cardiorrespiratória em mulheres idosas com sobrepeso do programa de atividade física no SESC de Nova Friburgo/ RJ/Brasil. Revista Motricidade, 4(1), 22-27.

Gomes, K. V. \& Zazá, D. C. (2009). Motivos de adesão à prática de Atividade Física em idosas. Revista Brasileira de Atividade Física \& Saúde, 14(2), 132138.

Guedes, T. A., Martins, A. B. T., Acorsi, C. R. L. \& Janeiro, V. (2005). Estatística descritiva, Projeto de ensino aprender fazendo estatística. Maringá: Universidade Estadual de Maringá.

Hauser, E., Martins, V. F., Teixeira, A. R. \& Gonçalves, A. G. (2015). ConScientiae Saúde, 14(2), 270-276. https://doi.org/10.5585/conssaude.v14n2.5512.

Heyward, V. H. \& Stolarczik, L. M. (2000). Avaliação da composição corporal aplicada. Manole.

Inouye, K. \& Pedrazzani, E. S. (2007). Nível de instrução, status socioeconômico e avaliação de algumas dimensões da qualidade de vida de octogenários. Revista Latino-Americana de Enfermagem, 15(spe), 742-747.

Leite-Cavalcanti, C., Rodrigues-Goncalves, M. C., Rios-Asciutti, L. S. \& Leite-Cavalcanti, A. (2009). Prevalência de doenças crônicas e estado nutricional em um grupo de idosos brasileiros. Revista de Salud Pública, 11(6), 865-877.

Li, A.M., Yin, J., Yu, C. C., Tsang, T., So, H. K. \& Wong, E, Chan, D., Hon, E. K. L. \& Sung, R. (2005). The six-minute walk test in healthy children: reliability and validity, Eur Respir J .25(6),1057-60.

Loureiro, N. S. L., Amaral, T. L. M., Amaral, C. A., Monteiro, G. T. R. M., Vasconcellos, M. T. L. \& Bortolini, M. J. S. (2020). Relação de indicadores antropométricos com fatores de risco para doença cardiovascular em adultos e idosos de Rio Branco, Acre. Revista de Saúde Pública, 54, 24. Epub March 16, 2020 .

Mazo, G. Z., Lopes, M. A. \& Benedetti, T.B. (2009). Atividade física e o idoso: concepção gerontológica. (3ª Ed.). Sulina.

Meurer, S. T., Beneditti, T. R. B. \& Mazo, G. Z. (2012). Fatores motivacionais de idosos praticantes de exercícios físicos: umestudo baseado na teoria da autodeterminação. Estudos de Psicologia (Natal), 17(2), 299-304. https://dx.doi.org/10.1590/S1413-294X2012000200014.

Morales-Blanhir, J. E., Vidal, C. D. P., Romero, M. J. R., Castro, M. M. G., Villegas, A. L. \& Zamboni, M. (2011). Teste de caminhada de seis minutos: uma ferramenta valiosa na avaliação do comprometimento pulmonar. Teste de caminhada de seis minutos: uma ferramenta valiosa na avaliação do comprometimento pulmonar. Jornal Brasileiro de Pneumologia, 37(1), 110-117. https://dx.doi.org/10.1590/S1806-37132011000100016.

Morgado, R. A. (2013). Atividade Física na Terceira Idade. Relatório de estágio profissional de Mestrado, Faculdade de Desporto, Universidade do Porto, Portugal.

Nunes, M. E. S. \& Santos, S. (2009). Avaliação funcional de idosos em três programas de atividade física: caminhada, hidroginástica e Lian Gong. Revista Portuguesa de Ciências do Desporto, 9(2-3), 150-159. 
Paschoal, S. M. P. (2004). Qualidade de vida do idoso: construção de um instrumento de avaliação através do método do impacto clínico. Tese de Doutorado, USP, São Paulo, SP, Brasil.

Ramos, L. R. (2009). Saúde Pública e envelhecimento: o paradigma da capacidade funcional. BIS. Boletim do Instituto de Saúde (Impresso), (47), $40-41$.

Ribeiro, F., Gomes, S., Teixeira, F., Brochado, G. \& Oliveira, J. (2009). Impacto da prática regular de exercício físico no equilíbrio, mobilidade funcional e risco de queda em idosos institucionalizados. Revista Portuguesa de Ciências do Desporto, 9(1), 36-42.

Ribeiro, R. M., Tribess, S., Santos, A. S., Pinto, L. L. T., Ribeiro, M. C. L., Roza, L. B. \& Virtuoso Júnior, J. S. (2015). Barreiras no engajamento de idosos em serviços públicos de promoção de atividade física. Ciência \& Saúde Coletiva, 20(3), 739-749. https://doi.org/10.1590/1413-81232015203.03702014.

Rikli, R. E. \& Jones, C. J. (1999). Development and validation of a functional fitness test for community-residing older adults. J Aging Phys Activity, 7(2), 129161.

Rikli, R. E. \& Jones, C. J. (2001). Senior Fitness Test Manual. Champaign, IL: Human Kinetics.

Rogers, M. E., Rogers, N. L. Takeshima, N. \& Islam, M. M. (2003). Methods to assess and improve the physical parameters associated with fall risk in older adults. Prev Med, 36(3), 255-64. https://doi.org/10.1016/S0091-7435(02)00028-2.

Silva, C. A. P. (2017). Avaliação do nível de aptidão física funcional em idosos praticantes de Ginástica Para Todos (GPT). Mais60 - Estudos sobre Envelhecimento, 28(69), 74-89.

Miyasike-da-Silva, V., Villar, R., Zago, A. S., Polastri, P. F. \& Gobbi, S. (2002). Nível de agilidade em indivíduos entre 42 e 73 anos: efeitos de um programa de atividades físicas generalizadas de intensidade moderada. Revista Brasileira de Ciências do Esporte, 23(3), 65-79.

Martins, R. J. (2016). Aptidão física, atividade física e qualidade de vida relacionada com a saúde de idosos. Dissertação de Mestrado, Escola Superior de Tecnologia da Saúde de Coimbra, Portugal.

Sousa, A. I., \& Silver, L. D. (2008). Perfil sociodemográfico e estado de saúde auto-referido entre idosas de uma localidade de baixa renda. Escola Anna Nery, 12(4), 706-716. https://dx.doi.org/10.1590/S1414-81452008000400015.

Squarcini, C. F. R. (2015). Programas de atividade física para idosos: avaliação da produção científica brasileira utilizando o modelo RE-AIM. Rev. Bras. Geriatr. Gerontol., 18 (4), 909-920.

Vila, C. P., Silva, M. E. M., Simas, J. P. N., Guimarães, A. C. A., \& Parcias, S. R. (2013). Aptidão física funcional e nível de atenção em idosas praticantes de exercício físico. Revista Brasileira de Geriatria e Gerontologia, 16(2), 355-364. https://doi.org/10.1590/S1809-98232013000200015.

Who. (1995). World Health Organization. Physical Status: The Use and Interpretation of Anthropometry. Technical Report Series 854. Geneva: WHO.

Who. (2011). World Health Organization. Global recommendations on physical activity for health. Geneva: WHO.

Who. (2005). World Health Organization. Organização Mundial de Saúde. Envelhecimento ativo: uma política de saúde. Brasília: Organização Pan-Americana da Saúde.

Who. (2000). World Health Organization. Obesity: preventing and managing the global epidemic. Geneva: WHO.

Xavier, F. Xavier, F., M. P., Bisol, L. W., Fernandes, D. D., Schwanke, C. \& Moringuchi, E. H.. (2000). Octagenários de Veranópolis: as condições psicológicas, sociais e de saúde geral de um grupo representativo de idosos com mais de 80 anos residentes na comunidade. Rev AMRIGS, 44(1/2), 25-9. 